\title{
Superior capsule reconstruction versus Reverse shoulder arthroplasty in massive rotator cuff tears: A comparative study
}

Satoru Ohta ( $\sim$ ootasato@shinseikai.or.jp )

Shinseikai Toyama Hospital

Yoshiyuki Ueda

Shinseikai Toyama Hospital

Osamu Komai

Shinseikai Toyama Hospital

\section{Research Article}

Keywords: Massive rotator cuff tear, Superior capsule reconstruction, Reverse shoulder arthroplasty, downward external rotation and internal rotation, Japanese

Posted Date: April 6th, 2022

DOI: https://doi.org/10.21203/rs.3.rs-1348181/v2

License: (9) This work is licensed under a Creative Commons Attribution 4.0 International License. Read Full License 


\section{Abstract}

Introduction: Superior capsule reconstruction (SCR) was developed in Japan in 2010 as a surgical treatment strategy for massive rotator cuff tears, This study was aimed at performing a comparative analysis of the postoperative results for SCR versus reverse shoulder arthroplasty (RSA), two surgical approaches currently used to treat massive rotator cuff tears.

Materials and Methods: Patients who underwent surgeries from 2014 to 2018 and were followed up for at least 2 years were included in the study. Subsequently, the cases of Hamada classifications 1-3 were retrospectively targeted. SCR comprised 38 patients (mean age 71.4 years) and RSA comprised 24 patients (mean age 78.9 years). The Japanese Orthopaedic Association (JOA) score, University of California at Los Angeles (UCLA) score, active elevation angle, and downward external rotation and internal rotation angles before and after the surgery were evaluated and compared between the groups.

Results: In postoperative group comparison (observed value), SCR significantly improved from RSA in terms of JOA score $(p=0.0060)$, UCLA score $(p=0.0042)$, elevation angle $(p=0.0070)$, downward external rotation $(p<0.001)$, and internal rotation $(p<0.001)$. In terms of postoperative comparison between groups (amount of change), RSA was superior in terms of JOA score $(p=0.0128)$ and UCLA score $(p=0.0055)$ and SCR showed improvement in terms of downward external rotation $(p<0.001)$ and internal rotation $(p=0.0016)$.

Conclusions: The results of treatment of massive rotator cuff tears in patients with Hamada's classification 1-3 were good for both techniques. In the postoperative comparison between the groups, downward external rotation and internal rotation showed a significant improvement in SCR in both the observed values and the amount of change.

\section{Introduction}

For large-to-massive tears that are difficult to repair, various surgical methods have been used, including debridement [1], partial repair [2], patch grafting [3-5], superior capsule reconstruction [6-9], muscle tendon transfers (that involve musculus latissimus dorsi [10], pectoralis major, trapezius, and teres minor), artificial joint procedures (such as the reverse type [11, 12]), artificial humeral head replacement (small head diameters), and muscle tendon transfers [13]. However, obtaining acceptable outcomes using any of these surgical methods can be challenging, and symptoms such as functional impairment and pain often remain after surgery. Among the available techniques, we have been using superior capsular reconstruction (SCR) and reverse shoulder arthroplasty (RSA) as the main surgical methods for cases of rotator cuff tears that are difficult to repair.

The SCR is a joint-preserving surgery developed in Japan by Mihata et al. [9] in 2010. The use of grafts, such as the fascia lata, to achieve anatomical reconstruction is a relatively new surgical technique that is gaining popularity owing to its biomechanical efficacy and excellent early clinical results. This joint- 
preserving procedure improves the biomechanical stability of the shoulder; however, it is a technically difficult procedure that requires extended surgical time. Also, postoperative rehabilitation takes time.

The RSA was introduced in Japan in 2014. Massive rotator cuff tear and cuff tear arthroplasty (CTA) in the elderly present with pseudoparalysis, and RSA is an effective treatment for these disorders. RSA that is currently performed utilizes a semi-constrained type artificial joint developed by Paul Grammont in 1986. In this surgical technique, the center of rotation (COR) of the scapulohumeral joint is moved inward and downward of the CTA [14]. It is said that the action of the deltoid muscle is increased by lowering the $\mathrm{COR}$, and it becomes possible to effectively raise the upper limb. RSA is expected to improve shoulder joint function in the early postoperative period and can be said to be a useful treatment for CTAs with pseudoparalyzed shoulders in the elderly with massive rotator cuff tears. However, because of the nonanatomical structure and insufficient long-term reports, some unclear points remain in the clinical course. Normal/full active range of motion of the shoulder joint following RSA is not expected.

We have performed SCR or RSA surgery on patients with massive rotator cuff tears and CTA. However, there are few comparative papers on these two techniques. Hence, this study intended to compare the postoperative results of SCR and RSA and to evaluate the indications for surgery in case of massive rotator cuff tears that are difficult to repair, although the concept of each surgery is different (Fig. 1).

\section{Methods}

\section{Target population and Methods}

This retrospective study was approved by the Institutional Review Board of our institution. All patients provided informed consent for this study. SCR (S group) was performed from 2011 to 2018, and RSA (R group) was performed by the same surgeon for massive rotator cuff tears for which primary repair was difficult from 2014 to 2018. The S group consisted of 38 patients (22 males and 16 females) and the R group consisted of 24 patients (11 males and 13 females) (Table 1). In this study, we targeted cases of Hamada classification [15] 1-3. Patients with follow-up data for at least 2 years were included. 
Table 1

Patient characteristics

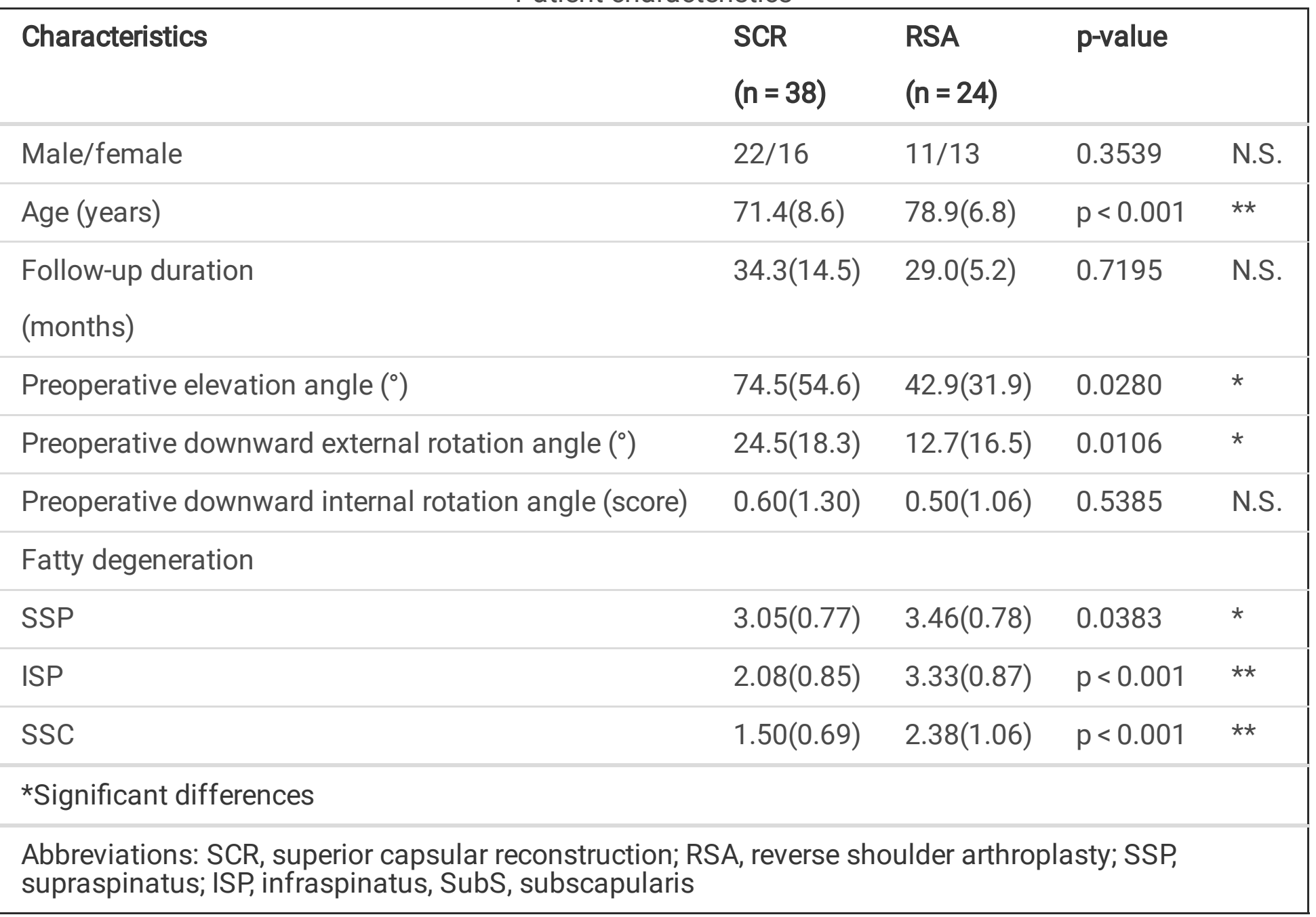

\section{Surgery}

Surgery was performed with general anesthesia, and an interscalene block was given to all patients. SCR was performed using the lateral decubitus traction technique (two positions). Arthroscopic mobilization of the rotator cuff was done to confirm the difficulty in pulling the tendon toward the greater tuberosity, and the size of the rotator cuff tear was measured. The fascia lata was harvested as a graft from the disinfected ipsilateral proximal lateral thigh. The grafts were created in duplicate and were $6-8 \mathrm{~mm}$ in thickness(Fig. 2a.b). The graft is guided into the joint through a 10cc syringe (Fig. 2c).

In accordance with the surgical method reported by Mihata et al. [6, 9], a suture-bridge method was adopted using two 4.5-mm suture anchors (Cork screw ${ }^{\circledR}$ FT, Arthrex Japan G.K., Tokyo) on the medial side of the suprascapular nodule and FiberTape ${ }^{\circledR}$ (Arthrex, Naples) or ULTRA Tape (Smith \& Nephew, Andover) on the medial side of the greater tuberosity. Posteriorly, lateral suturing of the infraspinatus tendons and grafts was performed with FiberWire (Arthrex, Naples FL). The subscapularis tendon was repaired if there was a tear. 
RSA was performed in patients $\geq 70$ years of age and had a rotator cuff tear for which primary repair was difficult and who presented with an elevation difficulty of $\leq 100$ degrees according to the Japanese Orthopaedic Association (JOA) guidelines of 2014. In all patients, the deltoid-pectoralis major intermuscular approach was used. The model used for the Grammont type was Aequalis ${ }^{\text {TM }}$ Reversed (Tornier, France) in one patient, SMR ${ }^{\mathrm{TM}}$ Reverse (Lima, Corporate, Italy) in four, and DELTA XTEND ${ }^{\mathrm{TM}}$ (DePuySynthes Institute, USA) in six. For the lateralized type, the Trabecular Metal ${ }^{\mathrm{TM}}$ Reverse Shoulder System (Zimmer Biomet, USA) was used in four patients, the Comprehensive ${ }^{\circledR}$ Reverse Shoulder System (Zimmer Biomet, USA) in three, and the Trabecular Metal ${ }^{\mathrm{T} M}-$ Comprehensive $^{\circledR}$ mix use in six patients.

Postoperative rehabilitation was performed in accordance with the rehabilitation program for massive and extensive tears at our institution for both $\mathrm{S}$ group and $\mathrm{R}$ group. The patient was immobilized with a shoulder abduction brace for 3 weeks after the surgery, and hand and elbow exercises were started on the second postoperative day. Shoulder passive range of motion (ROM) training (scapular plane elevation) was performed from 3 weeks after the surgery, and internal/external rotation started from 4 weeks; however, internal/external rotation exercises were not performed for the $\mathrm{R}$ group. The pillows of the abduction brace were removed at 6 weeks, active assisted exercises were allowed $6-7$ weeks after the surgery, active exercises were allowed 8-9 weeks, and resistance exercises were allowed 12 weeks after the surgery.

\section{Assessment parameters}

Preoperatively and at the final postoperative follow-up, the JOA score, UCLA score, active elevation angle before and after surgery, downward external rotation angle, and internal rotation angle were quantified based on intermittent vertebral levels (S-0, L5-1, L4-2, L3-3, L2-4, L1-5, Th12-6), and comparisons were made before and after the surgery. Additionally, the following parameters were compared with the preoperative evaluation: number of patients who had pseudoparalysis $\left(<90^{\circ}\right)$, degree of muscle fatty degeneration, and Hamada grading. Postoperative re-tears of the SCR group were assessed by magnetic resonance imaging (Sugaya classification [16]). Type 4 and 5 were considered re-tears. Between-group comparisons by UCLA scores were performed to assess patient satisfaction. Complications during and after the surgery were also evaluated.

\section{Statistical analyses}

For statistical studies, Mann-Whitney's U test was used for patient background and postoperative group comparisons (observed values), and Wilcoxon's signed rank test was used for pre- and postoperative comparisons. Analysis of covariance was used for postoperative group comparisons (amount of change), and a significance level of $<5 \%$ was considered to show a significant difference.

\section{Results}

There were 38 patients ( 22 males and 16 females) in the S group with a mean age of $71.4 \pm 8.6$ (standard deviation, SD) years at the time of surgery, follow-up of $24-76$ months, and a mean \pm SD follow-up of 
$34.3 \pm 14.5$ months. There were 24 patients ( 11 males and 13 females) in the $R$ group with a mean \pm SD age of $78.9 \pm 6.8$ years at the time of surgery, follow-up of $24-48$ months, and a mean \pm SD follow-up of $29.0 \pm 5.2$ months (Table 1). The JOA score significantly improved in both groups from the preoperative mean \pm SD of $59.8 \pm 10.6$ points to the postoperative mean \pm SD of $86.0 \pm 6.5$ points in the $S$ group $(p<$ 0.001 ) and from the preoperative mean of $50.7 \pm 16.1$ points to the postoperative mean \pm SD of $80.1 \pm 9.7$ points in the R group $(p<0.001)$. The UCLA score improved significantly in both groups from the preoperative mean $\pm S D$ of $19.2 \pm 3.8$ points to the postoperative mean $\pm S D$ of $30.3 \pm 2.4$ points in the $S$ group $(p<0.001)$ and from the preoperative mean \pm SD of $16.5 \pm 4.3$ points to the postoperative mean \pm SD of $28.1 \pm 3.3$ points in the $R$ group $(p<0.001)$ (Table 2$)$.

Table 2

Comparison before and after surgery in both groups

\section{SCR}

\begin{tabular}{|c|c|c|c|c|}
\hline & Pre.Op. & Post.Op. & p-value & \\
\hline JOA & $59.8(10.6)$ & $86.0(6.5)$ & $\mathrm{p}<0.001$ & ** \\
\hline UCLA & $19.2(3.8)$ & $30.3(12.4)$ & $\mathrm{p}<0.001$ & ** \\
\hline Elevation angle $\left(^{\circ}\right)$ & $74.5(53.9)$ & $131.7(41.7)$ & $p<0.001$ & ** \\
\hline Downward external rotation angle $\left(^{\circ}\right)$ & $24.5(18.4)$ & $35.1(18.8)$ & 0.0034 & ** \\
\hline Downward internal rotation angle (score) & $0.60(1.3)$ & $1.40(1.2)$ & 0.0026 & ** \\
\hline \multicolumn{5}{|l|}{ RSA } \\
\hline & Pre.Op. & Post.Op. & p-value & \\
\hline JOA & $50.7(16.1)$ & $80.1(9.7)$ & $\mathrm{p}<0.001$ & ** \\
\hline UCLA & $16.5(4.3)$ & $28.1(3.3)$ & $\mathrm{p}<0.001$ & ** \\
\hline Elevation angle $\left(^{\circ}\right)$ & $42.9(33.3)$ & $114.2(26.1)$ & $\mathrm{p}<0.001$ & ** \\
\hline Downward external rotation angle $\left(^{\circ}\right)$ & $12.7(12.3)$ & 11.7(11.0) & 0.876 & N.S. \\
\hline Downward internal rotation angle (score) & $0.50(1.2)$ & $0.60(0.9)$ & 0.484 & N.S. \\
\hline
\end{tabular}

The active elevation angle improved from $74.5 \pm 53.9$ preoperatively to $131.7 \pm 41.7$ postoperatively in the $S$ group and from a mean \pm SD of $42.9 \pm 33.3$ preoperatively to a mean of $114.2 \pm 26.1$ postoperatively in the $\mathrm{R}$ group, with both groups showing a significant difference $(p<0.001)$. At the final follow-up, the active elevation angles for the observed values were significantly greater in the $S$ group than in the $R$ 
group ( $<$ 0.001) (Table 3); however, no significant differences (amount of change) were observed between the two groups in pre- and postoperative group comparison (N.S.) (Table 4).

Table 3

Postoperative comparison between groups (observed value)

\begin{tabular}{|lllll|}
\hline Post.Op. & & & & \\
\hline & SCR & RSA & p-value & \\
\hline JOA & 86.0 & 80.1 & 0.0060 & $* \star$ \\
\hline UCLA & 30.3 & 28.1 & 0.0042 & $* \star$ \\
\hline Elevation angle $\left(^{\circ}\right)$ & 131.7 & 114.2 & 0.0070 & $* *$ \\
\hline Downward external rotation angle $\left(^{\circ}\right)$ & 35.1 & 11.7 & $p<0.001$ & $* *$ \\
\hline Downward internal rotation angle $($ score $)$ & 1.40 & 0.60 & $p<0.001$ & $* \star$ \\
\hline
\end{tabular}

Table 4

Postoperative comparison between groups (change in amount)

\begin{tabular}{|lcccc|}
\hline & SCR & RSA & p-value & \\
\hline JOA & 26.2 & 29.4 & 0.0128 & $*$ \\
\hline UCLA & 11.1 & 11.6 & 0.0055 & $* *$ \\
\hline Elevation angle $\left(^{\circ}\right)$ & 57.2 & 71.3 & 0.2775 & N.S. \\
\hline Downward external rotation angle $\left(^{\circ}\right)$ & 10.7 & -1.0 & $p<0.001$ & $* *$ \\
\hline Downward internal rotation angle $($ score) & 0.82 & 0.08 & 0.0016 & $* *$ \\
\hline
\end{tabular}

Conversely, the mean \pm SD downward external rotation angle improved significantly from $24.5 \pm 18.4$ preoperatively to $35.1 \pm 18.8$ postoperatively in the $S$ group $(p<0.001)$, while the mean $\pm S D$ angle of external rotation was not significantly different $(12.7 \pm 12.3$ before the surgery and $11.7 \pm 11.0$ after the surgery) in the R group (N.S.) (Table 2). At the last observation, the downward external rotation angle for the observed values was significantly higher in the $S$ group than in the R group $(p<0.001)$ (Table 3$)$, and a significant difference (amount of change) was also observed between the two groups before and after the surgery $(p<0.001)$ (Table 4$)$. The mean \pm SD internal rotation angle (quantitative value) improved significantly from $0.6 \pm 1.3$ before surgery to $1.4 \pm 1.2$ after surgery in the $S$ group $(p<0.01)$; however, a significant difference was not observed in the $R$ group $(0.5 \pm 1.2$ before the surgery and $0.6 \pm 0.9$ after the surgery) (N.S.). Overall, the $\mathrm{S}$ group was significantly different from the R group in terms of the last observation values $(p<0.001)$ (Table 3 ), and a significant difference (amount of change) was also observed between the two groups before and after the surgery $(p<0.001)($ Table 4$)$. 
According to the preoperative evaluation $\left(<90^{\circ}\right)$, the number of patients with pseudoparalysis was significantly different between the groups as 27 out of 38 patients in the S group and 23 out of 24 patients in the $\mathrm{R}$ group had pseuodoparalysis $(\mathrm{p}<0.001)$. Preoperatively, when compared with the $S$ group, the R group showed progressive fatty degeneration according to the Goutallier classification [17] in the supraspinatus (SSP), infraspinatus (ISP), and subscapularis (SSC) muscles (Table 1).

In terms of postoperative satisfaction UCLA scores, there were no significant differences between the groups (N.S.), with a mean score of $4.3 \pm 1.3$ (SD) in the S group and $4.4 \pm 1.2$ (SD) in the R group.

With regard to the complications, re-tears were observed in five $(13.2 \%)$ out of thirty-eight patients who underwent SCR, two (40\%) of whom were switched to RSA. Synovitis, which appeared to be caused by graft sutures, was observed in one patient (2.4\%), and none of the patients experienced infection or neuropathy. Two (8.3\%) patients experienced peri-implant fractures during RSA surgery and one $(3.1 \%)$ experienced postoperative acromial fracture. None of the patients experienced dislocation, infection, or neuropathy.

\section{Discussion}

SCR is a relatively new surgical method developed in Japan, which is performed as a joint-preserving surgery. The procedure has so far been associated with excellent biomechanical outcomes and has been used widely in many countries based on initial clinical results. In comparison, RSA was introduced in Japan after SCR, and there are only limited reports that clearly discuss the selection between these two surgical methods.

In 2011, a switch was made at our institution from arthroscopic patch surgery using fascia lata to SCR to treat massive and extensive rotator cuff tears for which primary repair is difficult. SCR is associated with numerous advantages such as low re-tear rates, optimal elevation angles, and indications $[18,19]$. For those who underwent SCR at our institution, re-tears were observed in $5(13 \%)$ out of 38 patients, which is almost equivalent to the reports from various authors [20,21]. However, the Hamada classification of grade $\geq 4$ is regarded as one of the high risk factors [22] and is considered to be inappropriate for SCR surgery. Therefore, in this study, we compared Hamada grade 1-3 cases with RSA.

RSA was introduced in Japan in 2014. In accordance with JOA guidelines, patients in whom primary repair is not feasible and in whom elevation restriction is severe (such as those with pseudoparalysis) are suitable candidates for RSA; however, the level of evidence for this method is grade $B$ [23]. Currently, many patients who would generally undergo SCR are now opting for RSA. Short-term results with RSA at our institution are similar to those reported by various authors in Japan $[20,24]$ in terms of improvements in JOA score and elevation angle.

Although improvements in active elevation angle with RSA were obtained in this study, no improvements in internal and external rotation before and after the surgery were noted, which is consistent with previous reports $[18,20]$. Although this time we used 11 types of Grammont type as well as 13 types of lateralized 
type, neither internal nor external rotation was improved in our case. Various methods [25-27] for lateralization of RSA for which internal and external rotation are expected have been reported, and there have also been reports on latissimus dorsi and teres major transfers to improve the lag in preoperative external rotation [28]. If adequate internal and external rotation cannot be obtained, it can substantially affect the activities of daily living, especially in older patients. Therefore the surgical method should be carefully selected.

In the R group, progressive fatty degeneration was observed in all preoperative SSP, ISP, and SSC (Table 1). Compared to the tendons that required primary repair, fatty degeneration of the remaining tendons was less predominant in both groups. It has been reported that grade 3 or 4 and higher progression in RSA affects external rotation and postoperative results [29]. For internal and external rotation, the effect of fatty degeneration on rotator cuffs for each surgical method when SCR and RSA are compared and the condition of fatty degeneration prior to surgery must be standardized, and further examination, including that of the teres minor, is necessary.

There are no clear criteria for choosing SCR over RSA to treat massive and extensive rotator cuff tears for which primary repair is difficult. Ogimoto et al. [18] reported that SCR is chosen for patients with relatively mild arthropathic changes at Hamada grade $\leq 3$, whereas RSA is generally chosen for arthropathic changes at Hamada grade $\geq 4$. Furthermore, potential candidates for each group must meet the surgical indications specified in the JOA guidelines. At our institution, the risk of re-tears with SCR was significantly higher for patients $\geq 80$ years of age, Hamada grade $\geq 4$, and male patients according to the multiple regression analysis [21]. Based on this finding, RSA was chosen first for the high-risk group. Long-term results for SCR have not yet been reported; however, SCR is known to be a joint preservation surgery in which anatomical reconstruction is performed with few complications. Improvements were also observed in one study in terms of postoperative internal and external rotation [6]. Since capsular ligament is the most affected tissue in shoulder-joint ROM, the belief is that the anatomical reconstruction of the superior joint capsule helps improve the internal and external rotation in addition to active elevation. In this study, in patients with re-tears, the switch to RSA was performed in two out of five patients. The surrounding tissue of the shoulder was anatomically preserved, and none of the tissues were impaired in a few cases involving residual suture anchors from the SCR. However, since patients with re-tears did not have improved active elevation in addition to internal and external rotation, measures to prevent re-tearing were considered necessary in SCR. Therefore, a meticulous evaluation of surgery indications is required for this high-risk group.

According to the JOA guidelines of 2019 for RSA, patients $\geq 65$ years of age are indicated. At present, SCR is the joint preservation surgery that appears to be the first choice for patients who engage in immense physical work, sports enthusiasts, and those aged $\geq 65$ years for maintaining a high quality of life. The complications are also minimal $[6,8,9,21]$. Among the choices, SCR seemed to be associated with limitations in patients with arthropathic changes associated with Hamada grade $\geq 4$, in elderly patients, and in those with shoulder pseudoparalysis. In these cases, RSA may be the procedure of choice. The postoperative complications of RSA included dislocation, infections, and loosening of the components. 
The 10-year overall survival was reported to be $89 \%$; however, reports suggest that caution should be exercised in performing RSA in young patients [27]. Additionally, there seems to be limitations associated with RSA in terms of optimal postoperative internal and external rotation and recovery. Based on the characteristics of both surgical procedures, SCR is advantageous for patients in whom extended rehabilitation is acceptable, and RSA is considered to be effective for elderly patients who have difficulty in coping with the extended rehabilitation.

Comparing the two surgical methods has been somewhat difficult since each procedure and the set of indications are completely different. As a treatment strategy for massive rotator cuff tears for which primary repair is difficult, the implementation of SCR and RSA in Japan is still quite novel; however, as more procedures are performed, a high level of evidence is accumulating, and it seems that further improvements and developments can be expected in the future.

\section{Limitations}

One limitation of this study is that it was retrospective in nature. In addition, both SCR and RSA groups had small sample sizes and short follow-up periods. Furthermore, the indication for SCR has changed since it became available in Japan in 2014 because of the increasing number of patients for whom RSA was chosen despite SCR being indicated for the initial surgery. For SCR, differences in graft sizes, presence or absence of subscapularis tendon repair, and the type used for RSA were observed. Other baseline factors such as differences in preoperative elevation angle, downward external angle, and presence of fatty degeneration might have influenced our results. In the future, evaluations are needed to identify standardized conditions for each surgical method.

\section{Conclusion}

Significant differences were observed in the amount of change in the postoperative results of SCR and RSA, except for the elevation angle. Determining which procedure is superior over the other is somewhat difficult since the two groups differed substantially in terms of surgical methods and indications. However, improvements in internal and external rotation following SCR seemed to be one of the noticeable benefits of choosing SCR over RSA.

\section{Abbreviations}

SCR: Superior capsule reconstruction

RSA: Reverse shoulder arthroplasty

JOA: Japanese Orthopaedic Association

UCLA: University of California at Los Angeles 
CTA: Cuff tear arthroplasty

COR: Center of rotation

ROM: Range of motion

SSP: Supraspinatus

ISP: Infraspinatus

SSC: Subscapularis

\section{Declarations}

Ethics approval and consent to participate: All procedures performed in human subjects studies. Participants must comply with the ethical standards of institutional and/or national research committees and the 1964 Helsinki declaration and subsequent amendments, or comparable ethical standards.

Ethical approval was received from the Shinseikai Toyama Hospital Research Ethics Review Committee (No. 190715-1). Informed consent was obtained from all individual participants included in the study.

Consent for publication: Not Applicable

Availability of data and materials: The datasets used and analyzed in this study are available from the corresponding author upon reasonable request.

Competing interests: The authors declare that they have no competing interest $\mathbb{X}$

Funding: This research was not specifically funded by any funding agency in the public, for-profit, or nonprofit sectors.

Authors' contributions: Yoshiyuki Ueda and Osamu Komai were responsible for technical assistance in surgery, especially graft preparation.

Acknowledgements: The authors thank Y. Ueda for providing technical assistance, Kamobayashi for the statistical analysis of numerous patients, and Crimson Interactive Pvt. Ltd. (Ulatus) for their assistance in manuscript translation and editing.

Availability of data and materials: The datasets used and/or analyzed during the current study are available from the corresponding author on reasonable request.

\section{References}

1. Melillo AS, Savoie FH, Field LD (1997) Massive rotator cuff tears: debridement versus repair. Orthop Clin North Am 28:117-124. https://doi.org/10.1016/s0030-5898(05)70269-8 
2. Kim SJ, Lee IS, Kim SH, Lee WY, Chun YM (2012) Arthroscopic partial repair of irreparable large to massive rotator cuff tears. Arthroscopy 28:761-768. https://doi.org/10.1016/j.arthro.2011.11.018

3. Barber FA, Burns JP, Deutsch A, Labbé MR, Litchfield RB (2012) A prospective, randomized evaluation of acellular human dermal matrix augmentation for arthroscopic rotator cuff repair. Arthroscopy 28:8-15. https://doi.org/10.1016/j.arthro.2011.06.038

4. Rhee SM, Oh JH (2017) Bridging graft in irreparable massive rotator cuff tears: autogenic biceps graft versus allogenic dermal patch graft. Clin Orthop Surg 9:497-505. https://doi.org/10.4055/cios.2017.9.4.497

5. Yoon JP, Chung SW, Kim JY, et al. (2016) Outcomes of combined bone marrow stimulation and patch augmentation for massive rotator cuff tears. Am J Sports Med 44:963-971. https://doi.org/10.1177/0363546515625044

6. Mihata T, Lee TQ, Watanabe C, et al. (2012) Clinical results of arthroscopic superior capsule reconstruction for irreparable rotator cuff tears. Arthroscopy 29:459-470.

https://doi.org/10.1016/j.arthro.2012.10.022

7. Mihata T, McGarry MH, Kahn T, Goldberg I, Neo M, Lee TQ (2016) Biomechanical role of capsular continuity in superior capsule reconstruction for irreparable tears of the supraspinatus tendon. Am $\mathrm{J}$ Sports Med 44:1423-1430. https://doi.org/10.1177/0363546516631751

8. Mihata T, McGarry MH, Kahn T, Goldberg I, Neo M, Lee TQ (2016) Biomechanical effect of thickness and tension of fascia lata graft on glenohumeral stability for superior capsule reconstruction in irreparable supraspinatus tears. Arthroscopy 32:418-426. https://doi.org/10.1016/j.arthro.2015.08.024

9. Mihata T, Watanabe C, Kinoshita M, Fukunishi K, Tsujimura T, Ohue M (2010) Superior capsular reconstruction in patients with rotator cuff tears irreparable through primary repair (in Japanese). Katakansetsu 34:451-453. https://doi.org/10.11296/katakansetsu.34.451

10. El-Azab HM, Rott O, Irlenbusch U (2015) Long-term follow-up after latissimus dorsi transfer for irreparable posterosuperior rotator cuff tears. J Bone Joint Surg Am 97:462-469. https://doi.org/10.2106/JBJS.M.00235

11. Rhee YG, Cho NS, Moon SC (2015) Effects of humeral component retroversion on functional outcomes in reverse total shoulder arthroplasty for cuff tear arthropathy. J Shoulder Elbow Surg 24:1574-1581. https://doi.org/10.1016/j.jse.2015.03.026

12. Werner BC, Wong AC, Mahony GT, et al. (2018) Clinical outcomes after reverse shoulder arthroplasty with and without subscapularis repair: the importance of considering glenosphere lateralization. $J$ Am Acad Orthop Surg 26:e114-e119. https://doi.org/10.5435/JAAOS-D-16-00781

13. Miyoshi N, Suenaga N, Oizumi N, Taniguchi N, Ito H. (2014) Rotator cuff reconstruction and humeral head replacement using smaller humeral prosthesis in cuff tear arthropathy patients under 70 years of age. Open J Orthop 4:263-272. https://doi.org/10.4236/ojo.2014.410043

14. Boileau P, Watkinson DJ, Hatzidakis AM, Balg F (2005) Grammont reverse prosthesis: design, rationale, and biomechanics. J Shoulder Elbow Surg 14:147S-161S. 
https://doi.org/10.1016/j.jse.2004.10.006

15. Hamada K, Fukuda H, Mikasa M, Kobayashi Y: Roentgenographic findings in massive rotator cuff tears. A long-term observation. Clin Orthop Relat Res, 1990; 254: 92-6. https://doi.org/10.1097/00003086-199005000-00014

16. Sugaya $\mathrm{H}$, Maeda K, MatsukiK MJ (2007) Repair integrity and functional outcome after arthroscopic double-row rotator cuf repair. A prospective outcome study. J Bone Jt Surg Am 89:953-960. https://doi.org/10.2106/JBJS.F.00512

17. Goutallier D, Postel J, Bernageau J, Lavau L, Voisin MC (1994) Fatty muscle degeneration in cuf ruptures. Pre-and postoperative evaluation by CT scan. Clin Orthop Relat Res 304:78-83. PMID: 8020238

18. Ohta S (2013) Experience of arthroscopic superior capsular reconstruction for irreparable rotator cuff tears (in Japanese). Katakansetsu 37:705-708. https://doi.org/10.11296/katakansetsu.37.705

19. Ohta $S$ (2016) Experience of superior joint capsule reconstruction for rotator cuff tears for which primary repair is difficult (in Japanese). JOSKAS 41:616-621.

20. Ogimoto S, Tsuruta T (2017) Review of postoperative results of reverse prosthesis replacement with arthroscopic superior capsular reconstruction for rotator cuff tears irreparable by primary repair (in Japanese). Katakansetsu 41:751-754. https://doi.org/10.11296/katakansetsu.41.751

21. Lim S, AlRamadhan H, Kwak JM, Hong H, Jeon IH (2019) Graft tears after arthroscopic superior capsule reconstruction (ASCR): pattern of failure and its correlation with clinical outcome. Arch Orthop Trauma Surg 139:231-239. https://doi.org/10.1007/s00402-018-3025-7

22. Ohta S, Komai O, Onochi Y (2020) Outcomes of superior capsule reconstruction for massive rotator cuff tears and risk factors for postoperative retear. Arch Orthop Trauma Surg 140:1319-1325. https://doi.org/10.1007/s00402-019-03316-2

23. Bedi A, Dines J, Warren RF, Dines DM (2010) Massive tears of the rotator cuff. J Bone Joint Surg 92:1894-1908. https://doi.org/10.2106/JBJS.I.01531

24. Nagase $Y$, Yano Y, Morishige M, Asai H, Yoshikawa K, Watanabe H (2016) Short-term results and intraoperative and early complications of reverse shoulder arthroplasty (in Japanese). Katakansetsu 40:716-720. https://doi.org/10.11296/katakansetsu.40.716

25. Greiner S, Schmidt C, Herrmann S, Pauly S, Perka C (2015) Clinical performance of lateralized versus non-lateralized reverse shoulder arthroplasty: a prospective randomized study. J Shoulder Elbow Surg 24:1397-404. https://doi.org/10.1016/j.jse.2015.05.041

26. Helmkamp JK, Bullock GS, Amilo NR, et al. (2018) The clinical and radiographic impact of center of rotation lateralization in reverse shoulder arthroplasty: a systematic review. J Shoulder Elbow Surg 27:2099-2107. https://doi.org/10.1016/j.jse.2018.07.007

27. Müller AM, Born M, Jung C, et al. (2018) Glenosphere size in reverse shoulder arthroplasty: is larger better for external rotation and abduction strength? J Shoulder Elbow Surg 27:44-52. https://doi.org/10.1016/j.jse.2017.06.002 
28. Boileau P, Chuinard C, Roussanne Y, Neyton L, Trojani C (2007) Modified latissimus dorsi and teres major transfer through a single delto-pectoral approach for external rotation deficit of the shoulder: as an isolated procedure or with a reverse arthroplasty. J Shoulder Elbow Surg. 16:671-682. https://doi.org/10.1016/j.jse.2007.02.127

29. Favard L, et al. (2011) Reverse prostheses in arthropathies with cuff tear: are survivorship and function maintained over time? Clin Orthop Relat Res 469:2469-2475. https://doi.org/10.1007/s11999-011-1833-y

\section{Figures}

a

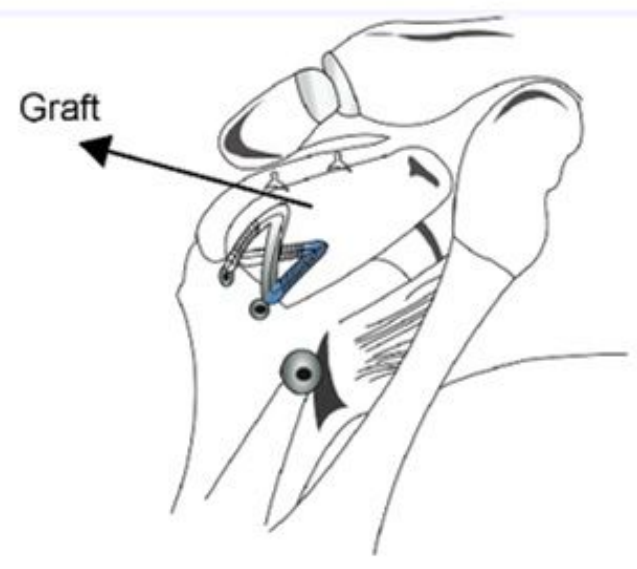

$\mathrm{b}$

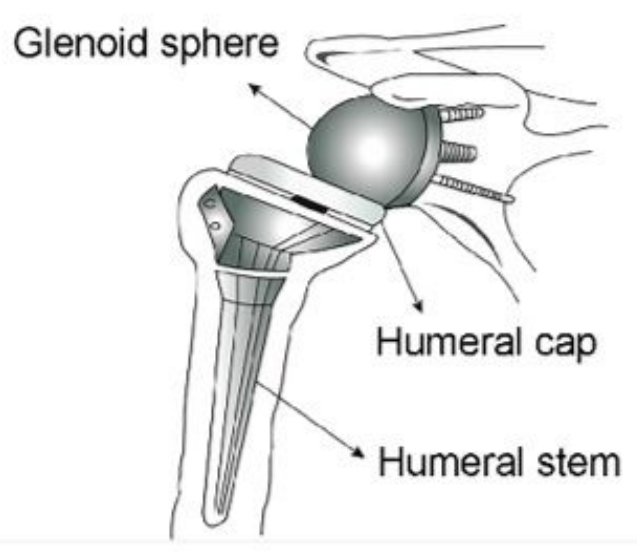

\section{Figure 1}

a Superior capsular reconstruction

b Reverse shoulder arthroplasty 


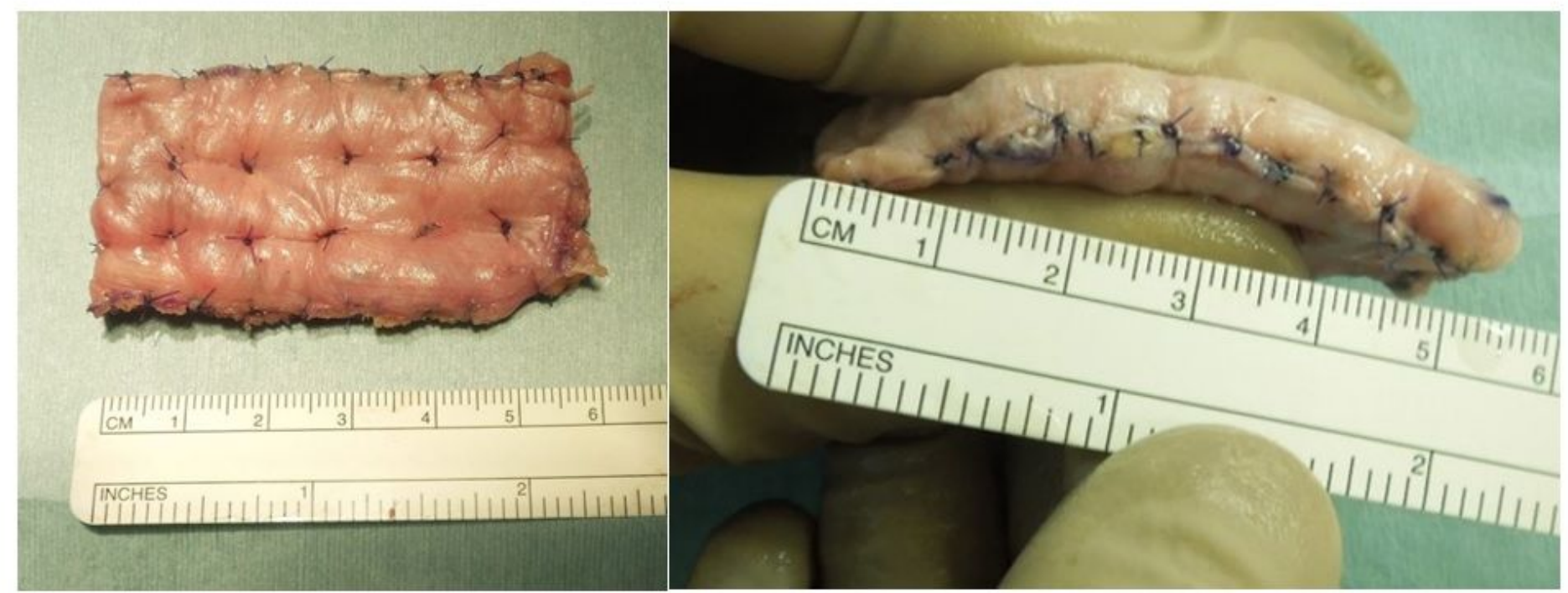

c

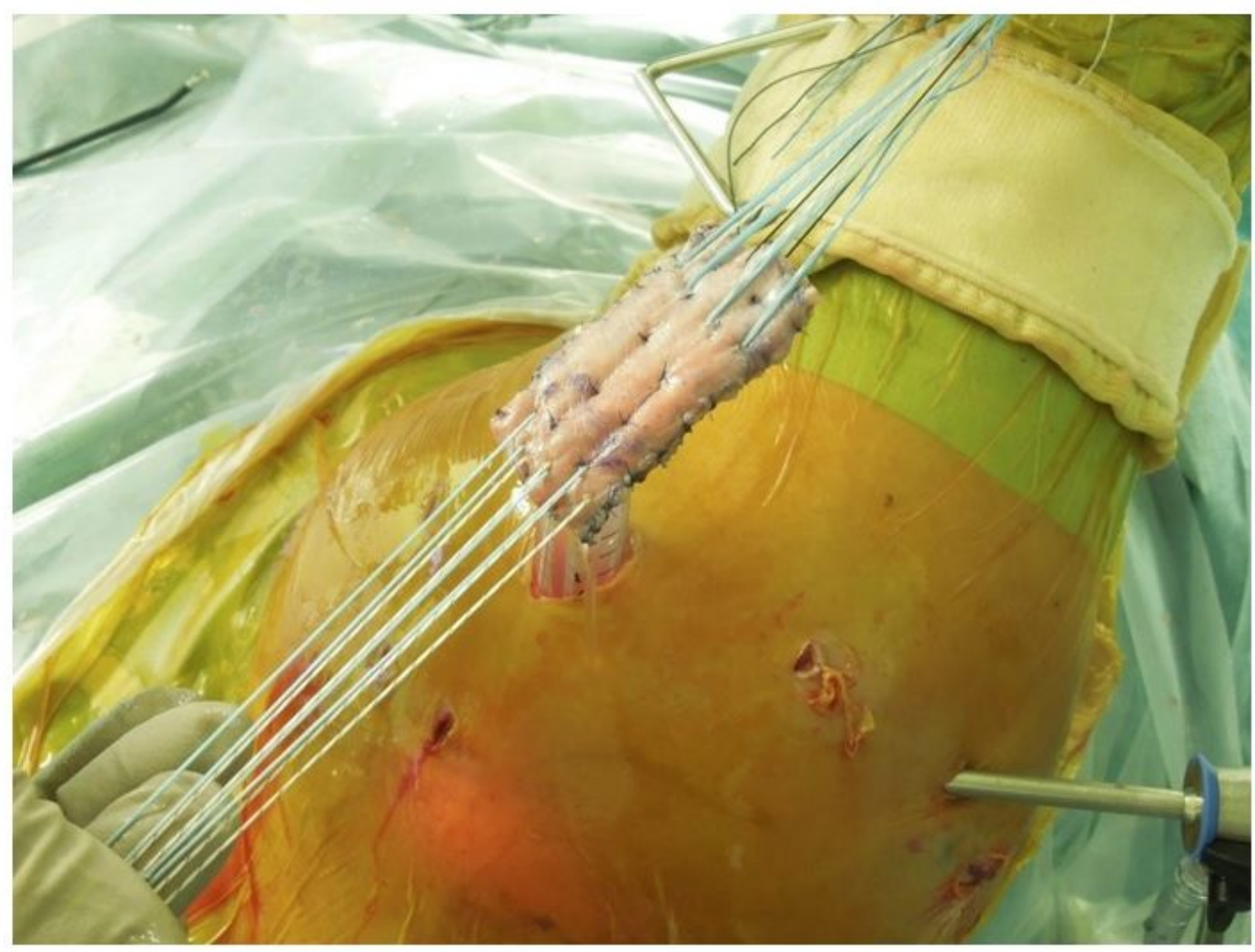

Figure 2

harvested fascia lata a $3 \times 6 \mathrm{~cm}$ in length and width

b 6-8 $\mathrm{mm}$ in thickness

c graft is guided into the joint through a 10cc syringe 